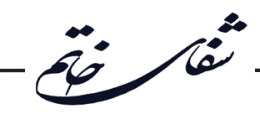

\title{
Investigating the Effect of Avicennia Marina Aqueous Extract on Neuronal Density of Hippocampal CA1, CA2, CA3 and Dentate Gyrus in Diabetic Rats
}

\author{
Seyed Damoon Sadoughi ${ }^{1 *}$, Mohammad Amin Edalatmanesh ${ }^{2}$, Raheleh Rahbarian ${ }^{3}$ \\ ${ }^{1}$ Young Researchers and Elite Club, Mashhad Branch, Islamic Azad University, Mashhad, Iran \\ ${ }^{2}$ Department of Physiology, College of Sciences, Shiraz Branch, Islamic Azad University, Shiraz, Iran \\ ${ }^{3}$ Department of Biology, College of Sciences, Payam-e-Noor University, Tehran, Iran
}

\begin{tabular}{lll}
\hline & Article Info: & \\
\hline Received: 29 Apr 2016 & Revised: 28 Jul 2016 & Accepted: 1 Sep 2016
\end{tabular}

\section{ABSTRACT}

Introduction: Chronic diabetes, via reduction of brain neurons, is associated with impaired learning and memory. According to the antioxidant effect of Avicennia marina, this study was conducted with the aim of investigating the neuroprotective effect of aqueous extracts of Avicennia marina on neuronal density in the hippocampal CA1, CA2, CA3, and dentate gyrus in diabetic rats. Materials and Methods: In this study 28 male rats were allocated into equal groups of control, diabetic, and treated diabetic 1 and 2 . The diabetes in diabetic and treated diabetic groups was induced using an intraperitoneal (ip) injection of alloxan. The treated diabetic groups 1 and 2 received the aqueous extract of Avicennia marina (ip, 100 and $200 \mathrm{mg} / \mathrm{kg}$, respectively) in alternate days for one month. Sterile distilled water was injected to the animals of control and diabetic groups. At the end of herbal treatments, animals were anesthetized and their brains were removed. After tissue processing, tissue sections were stained with Hematoxylin and Eosin. Then, the neuronal density in the hippocampal CA1, CA2, CA3, and dentate gyrus were investigated. Results: Treatment with Avicennia marina at $200 \mathrm{mg} / \mathrm{kg}$ in diabetic rats, but not $100 \mathrm{mg} / \mathrm{kg}$, prevented neuronal loss compared to non-treated diabetic group. Conclusion: Administration of Avicennia marina extract could be protect neurons against diabetes mediated cell degeneration.

Key words:

1. Hippocampus

2. Rats

3. Animals

*Corresponding Author: Seyed Damoon Sadoughi

E-mail: Damoon.sadoughi@mshdiau.ac.ir 


\title{
بررسى اثر عصارة آبى حرا بر دانسيتهُ نورونى CA1، CA2، CA3 و شكنج دندانهاى هييو كامٍ در موشهاى صحر اييى ديابتى نورنى
}

\author{
سيد دامون صدوقى"'، محمد امين عدالتمنش '، راهله رهباريان \\ 'باشَاه يُروهشَران جوان و نخبعان، واحد مشهد، دانشعاه آزاد اسلامى، مشهد، ايران \\ rَّروه فيزيولوزى، دانشكده علوم، واحد شير از، دانشكاه آزاد اسلامى، شيراز، ايران \\ "َكروه زيستشناسى، دانشكده علوم، دانشعاه يِيام نور، تهران، ايران
}

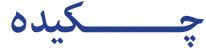

مقدمه: ديابت مزمن، از طريق كاهش نورونهاى مغزى با اختلال در يادگيرى و حافظه همراه است. با

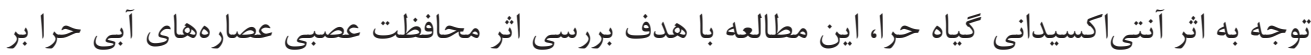

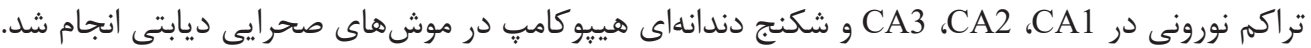

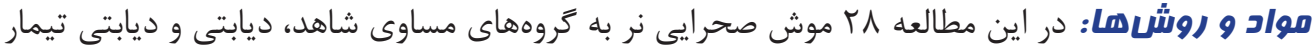

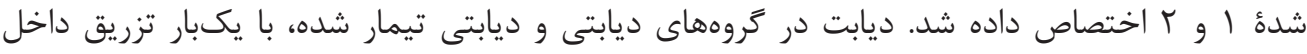

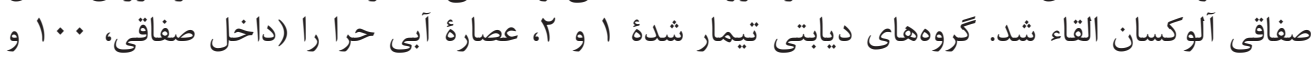

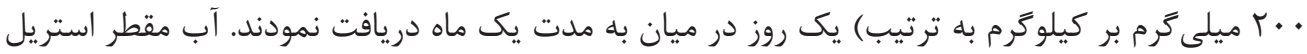

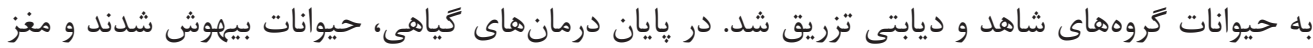

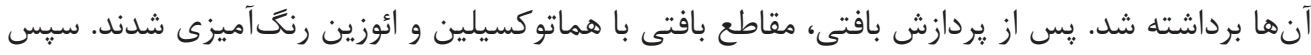

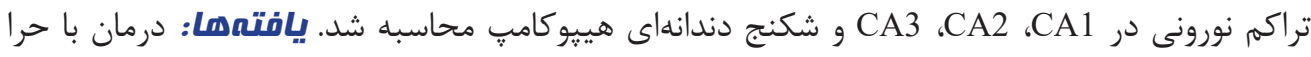

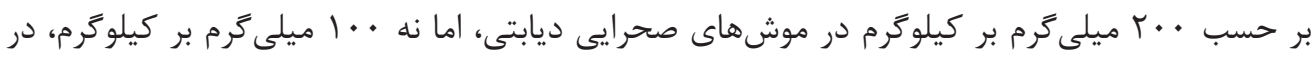

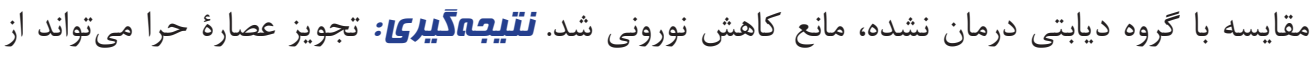

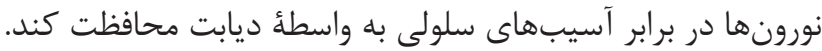

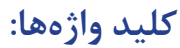
I.

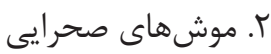

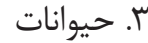

$$
\text { " نويسنده مسئول: سيد دامون صدوقى : }
$$

آدرس الكترونيكى: Damoon.sadoughi@mshdiau.ac.ir. 


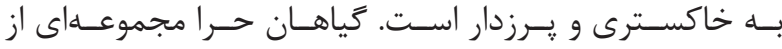

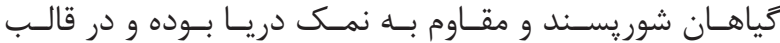

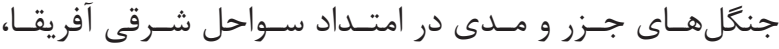

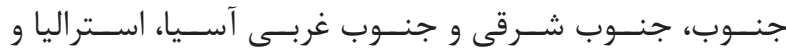

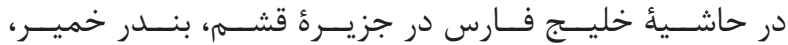

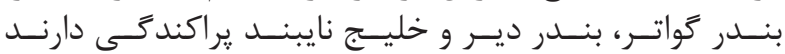

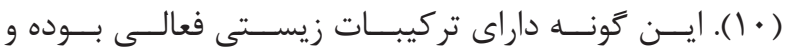

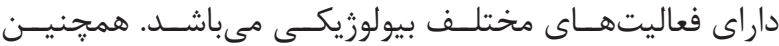

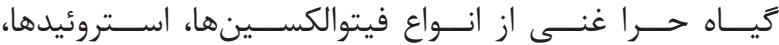

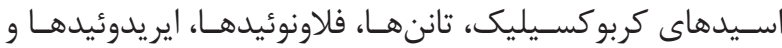

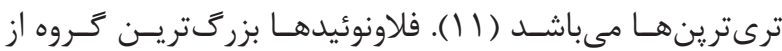

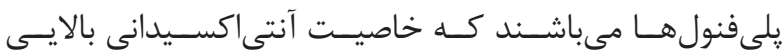

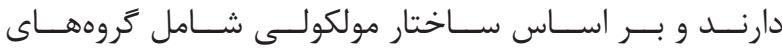

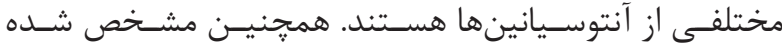

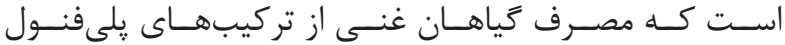

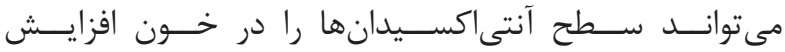

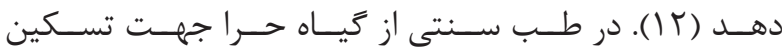

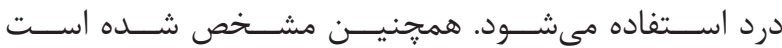

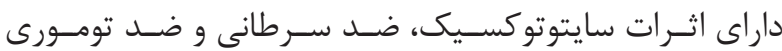

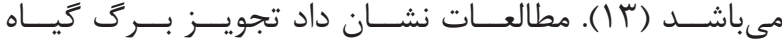

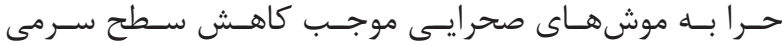

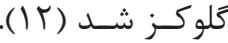

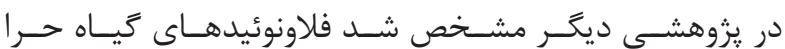

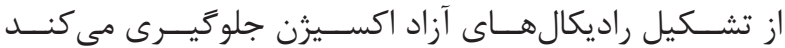

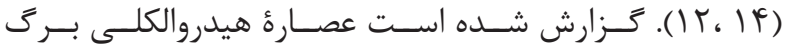

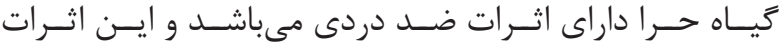

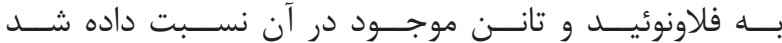

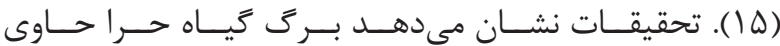

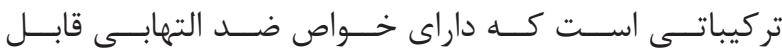

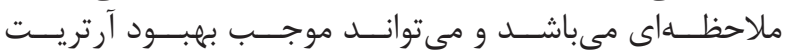

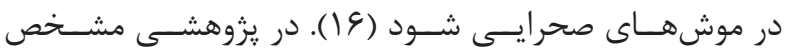

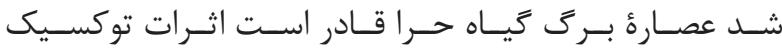

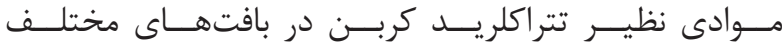

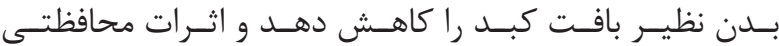

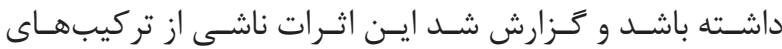

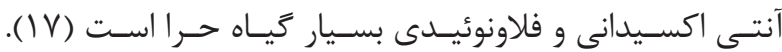

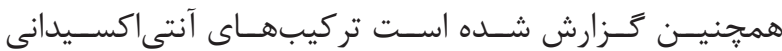

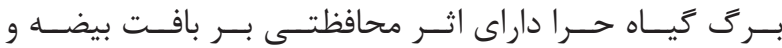

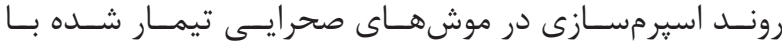

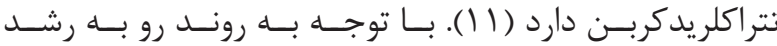

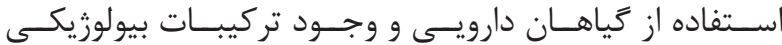

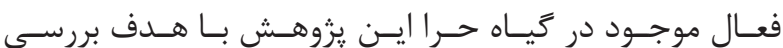

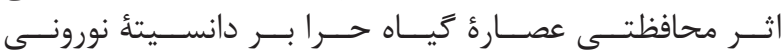

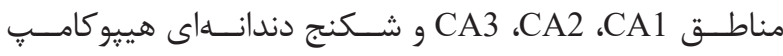

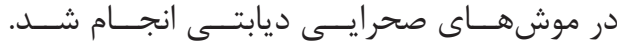

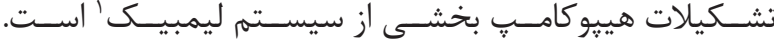

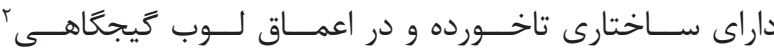

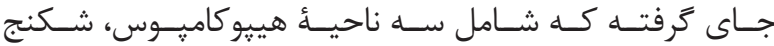

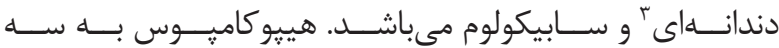

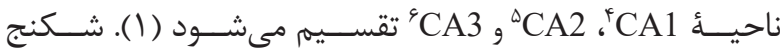

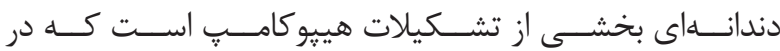

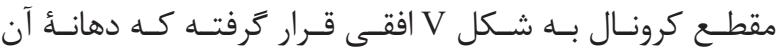

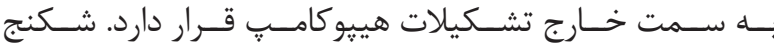

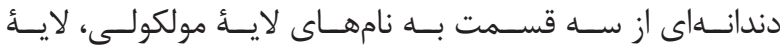

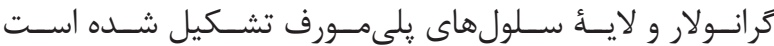

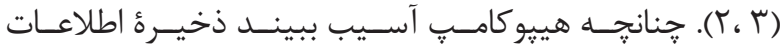

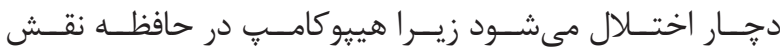

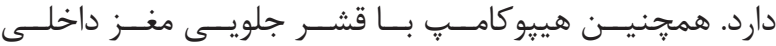

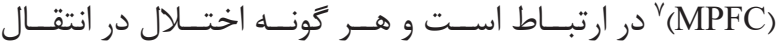

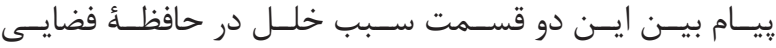

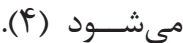

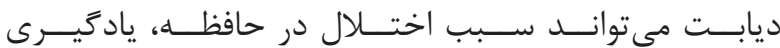

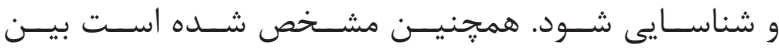

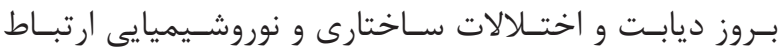

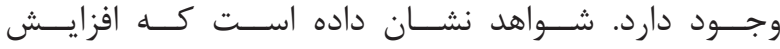

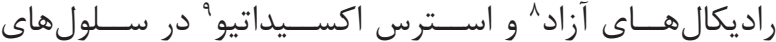

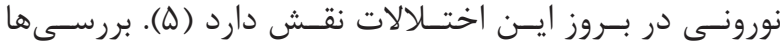

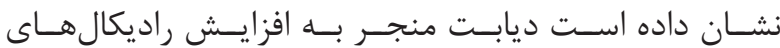

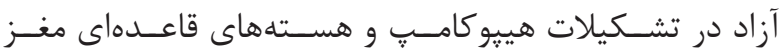

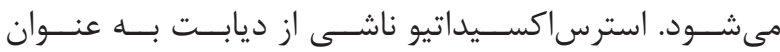

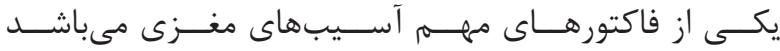

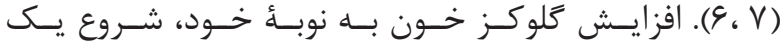

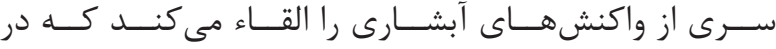

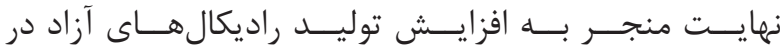

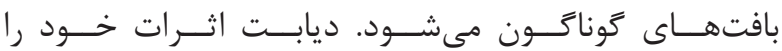

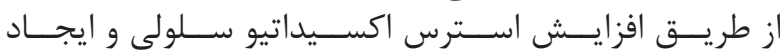

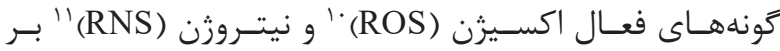

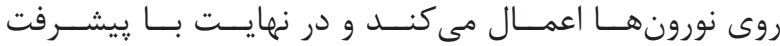

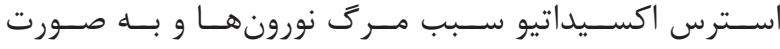

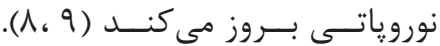
كيـاه حـرا بـا نـام علمسى Acennia marina (Forsk) Vierh

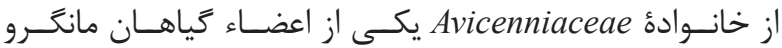

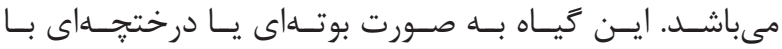

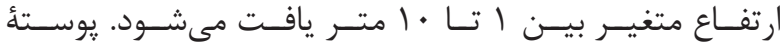

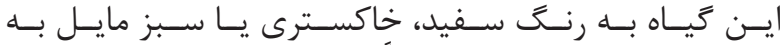

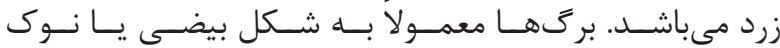

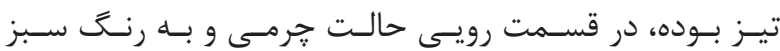

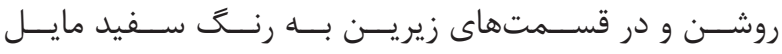

${ }^{7}$ Medial prefrontal cortex

${ }^{8}$ Free radicals

${ }^{9}$ Oxidative stress

${ }^{10}$ Reactive oxygen species

${ }^{11}$ Reactive nitrogen species 


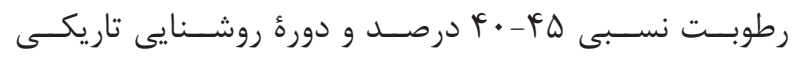

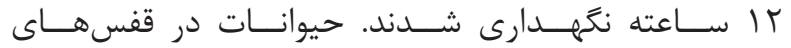

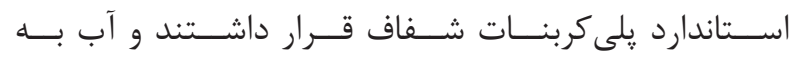

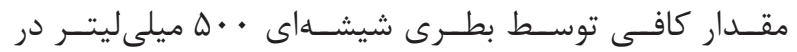

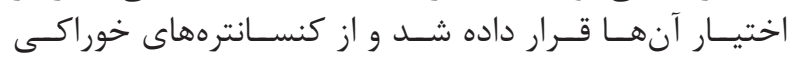

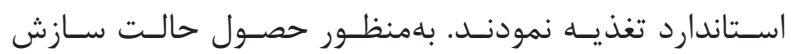

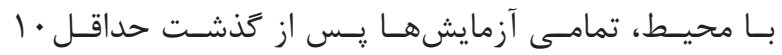

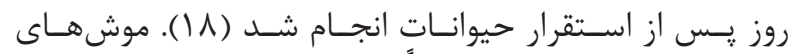

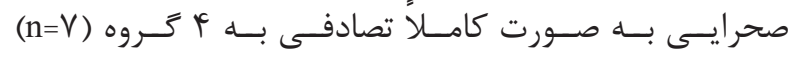

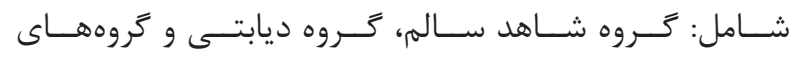

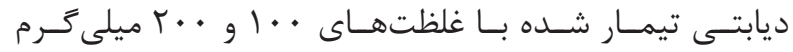

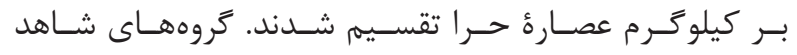

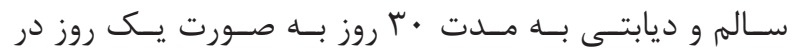

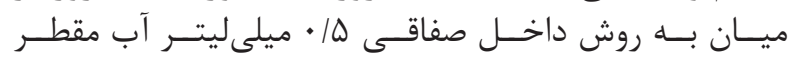

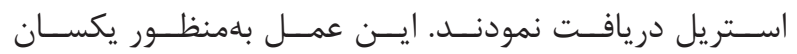

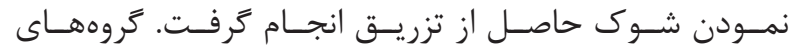

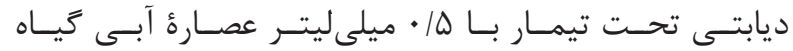

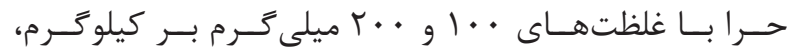

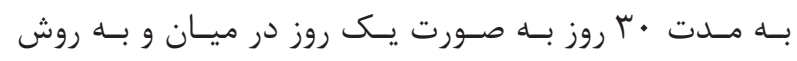

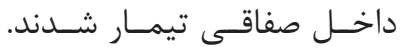

مطالعة بافتشناسى

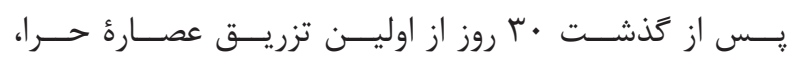

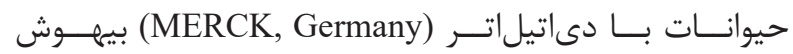

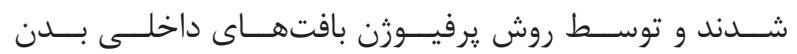

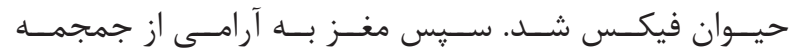

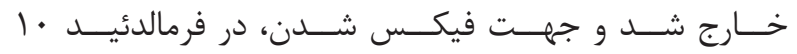

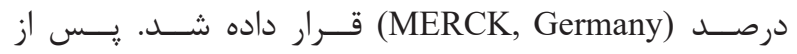

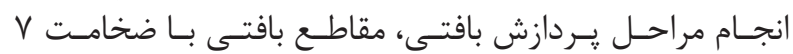

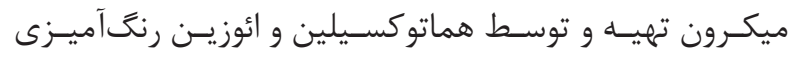

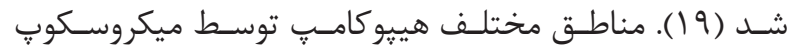

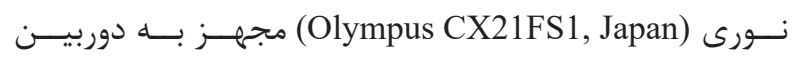

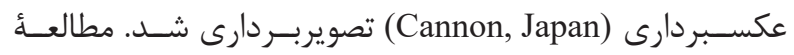

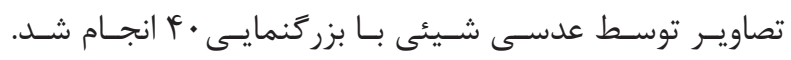
تراكسم نورونسى (N/mm³

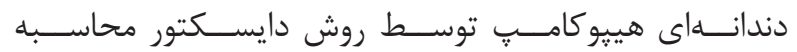

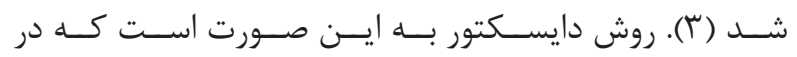

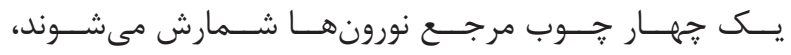

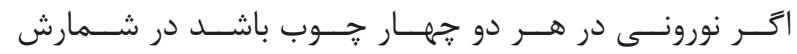

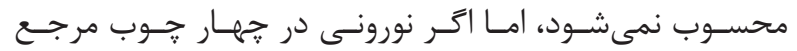

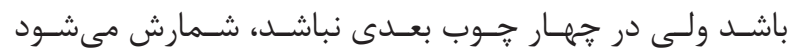

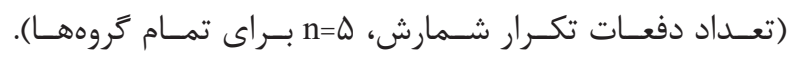

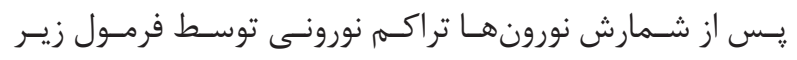

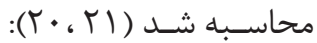

$\Sigma \mathrm{Q} / \Sigma$ frame $\times \mathrm{V}$ dissector $=\mathrm{ND}$

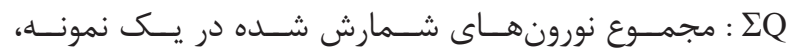

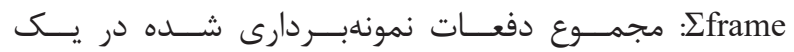

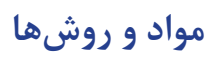

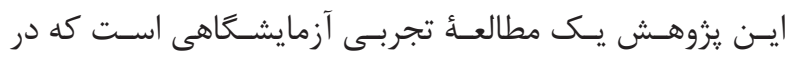

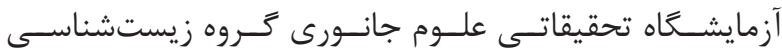

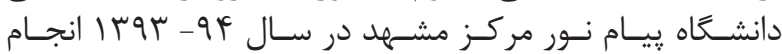

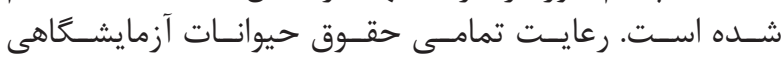

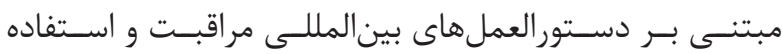

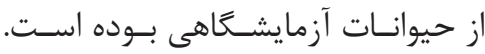
جمع آورى تياه و روش عصارهَيرى

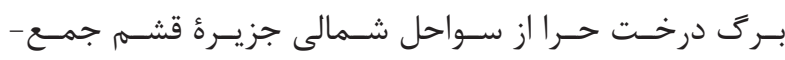

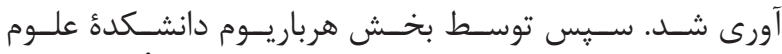

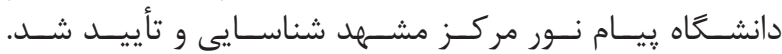

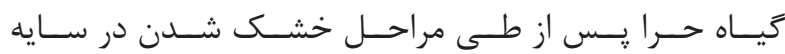

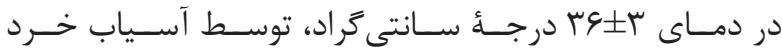

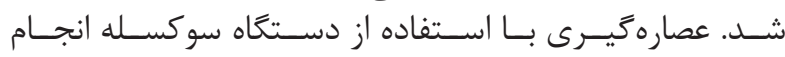

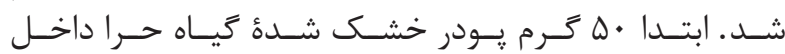

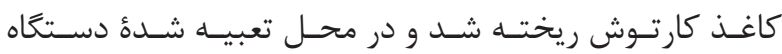

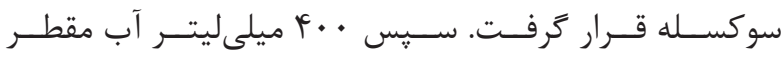

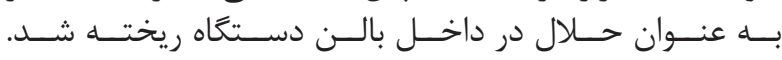

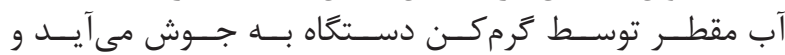

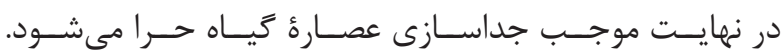

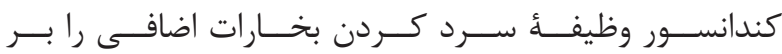

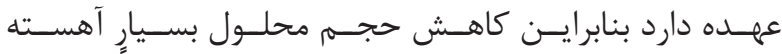

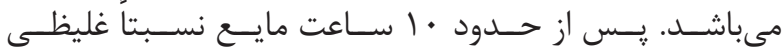

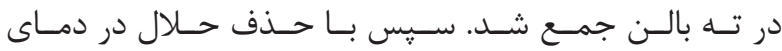

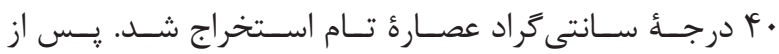

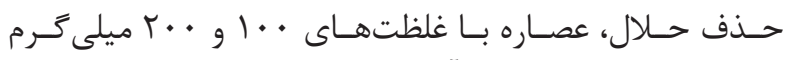

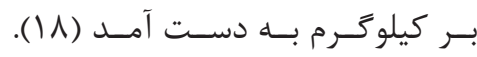

$$
\text { إيجاد هيبر كليسمى تجربى }
$$

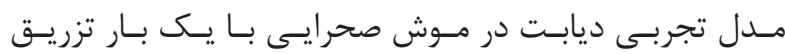

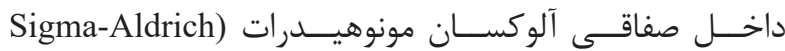
(Germany

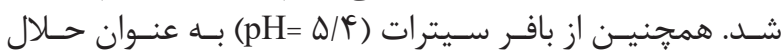

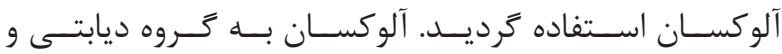

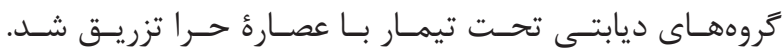

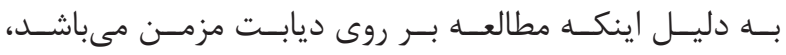

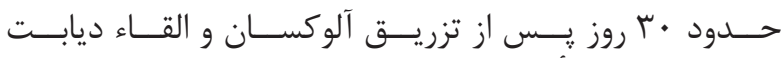

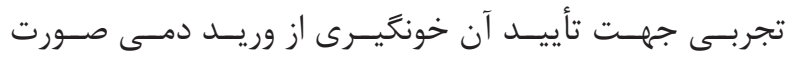

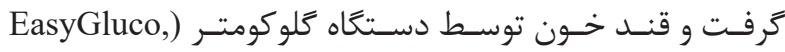
(Korea

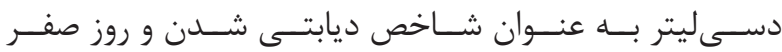

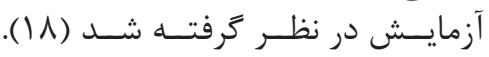

$$
\text { حيوانات و زروههاى مورد آزمايش }
$$

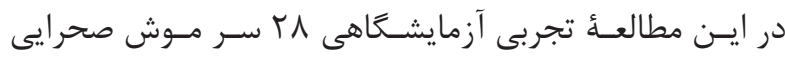

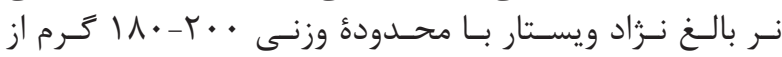

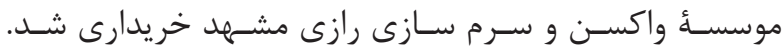

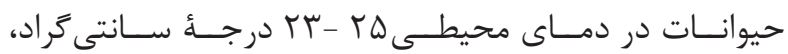




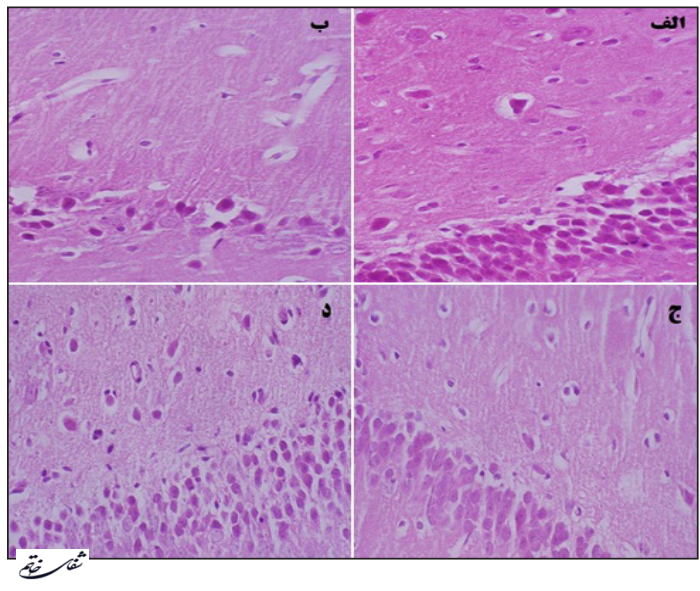

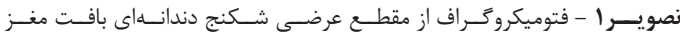

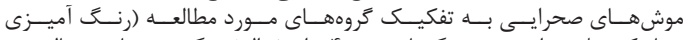

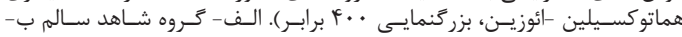

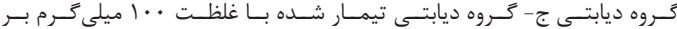

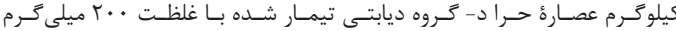

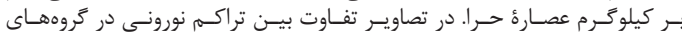

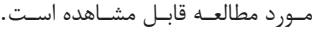

\section{بحث و نتيجه}

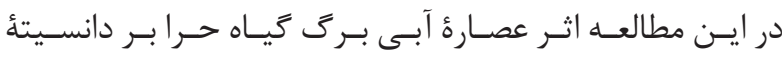

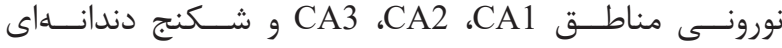

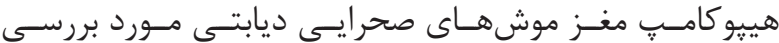

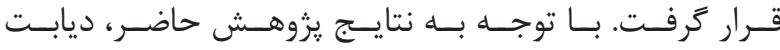

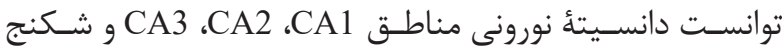

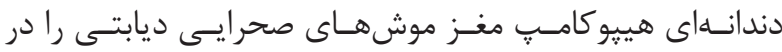

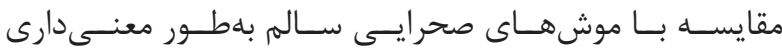

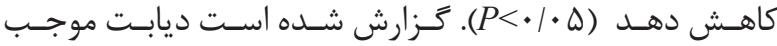

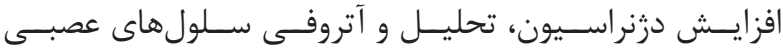

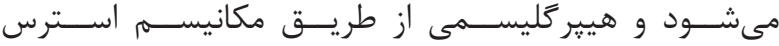

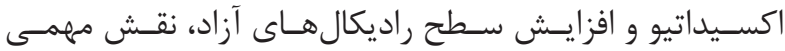

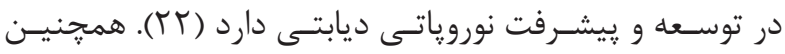

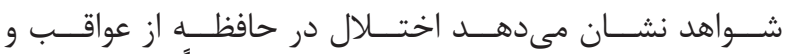

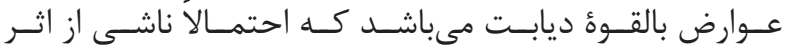

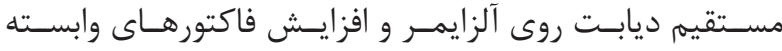

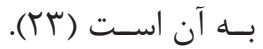

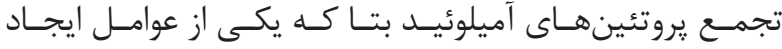

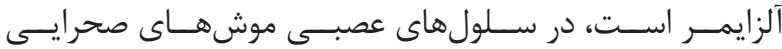

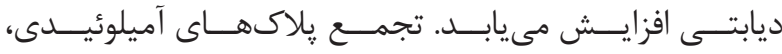

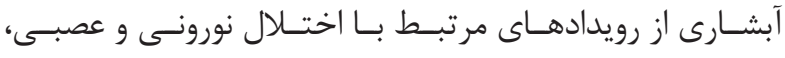

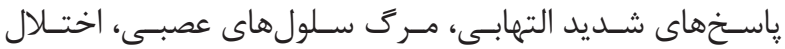

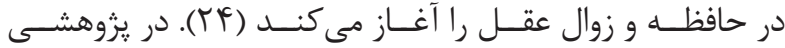

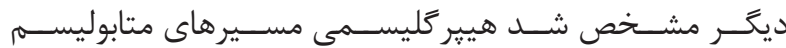

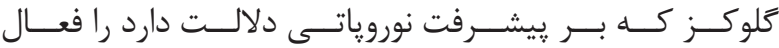

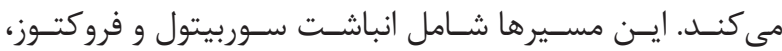

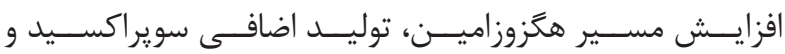

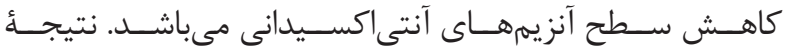

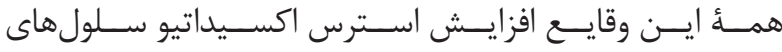

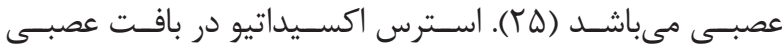

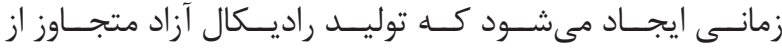

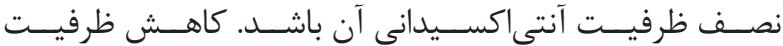

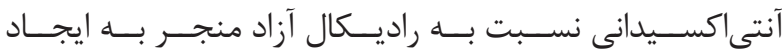

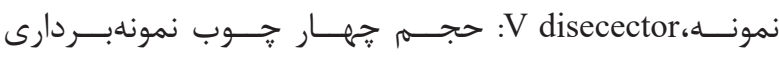

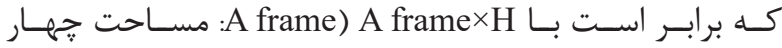

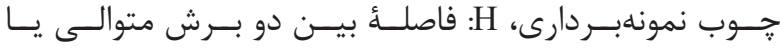

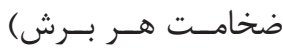

روش تجزيه و تحليل دادهها

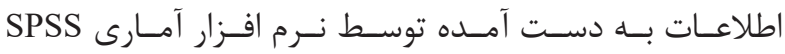

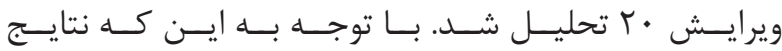

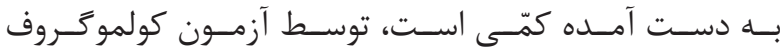

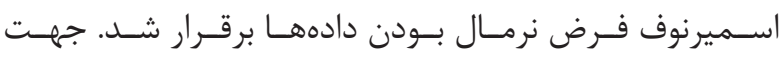

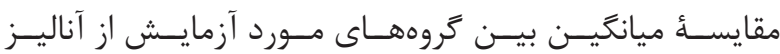

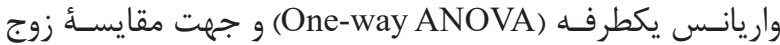

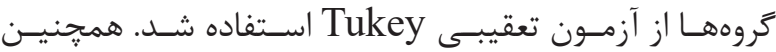

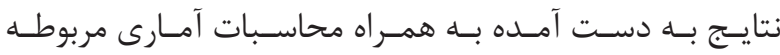

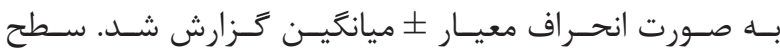

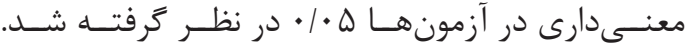

يافته ها

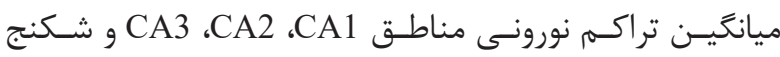

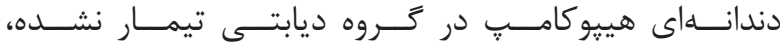

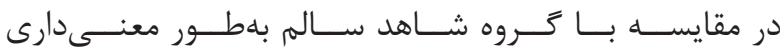

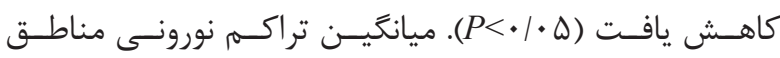

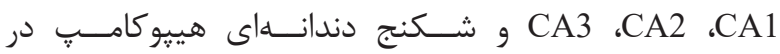

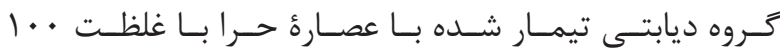

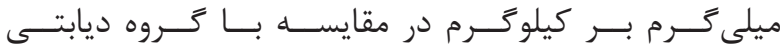

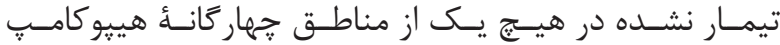

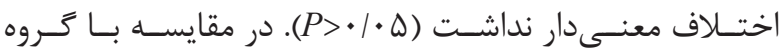

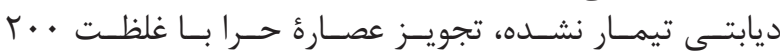

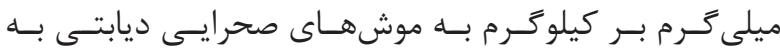

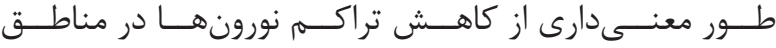

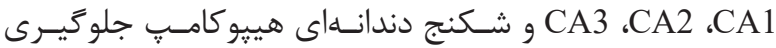

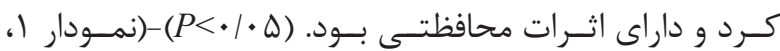
تصويسر (1) (1)

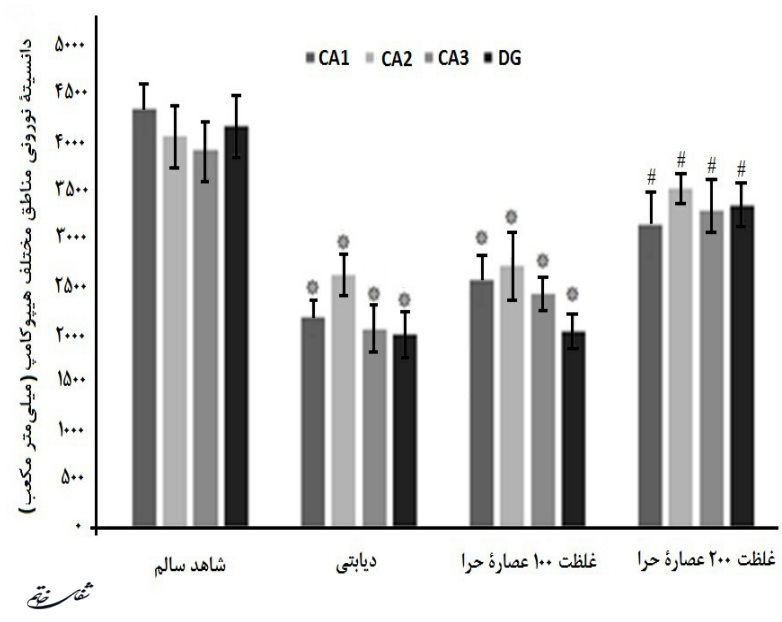

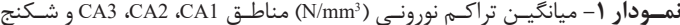

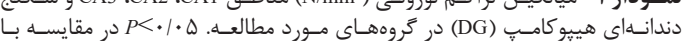

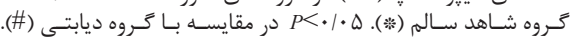




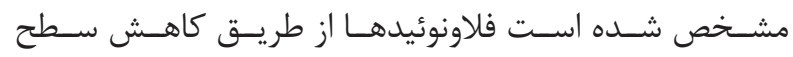

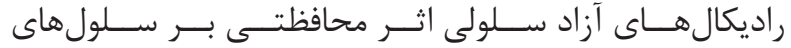

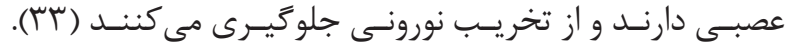

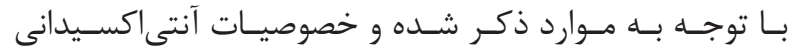

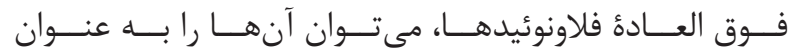

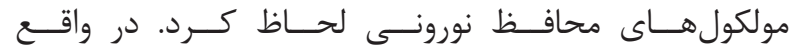

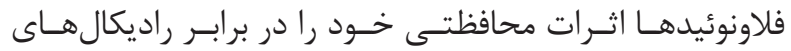

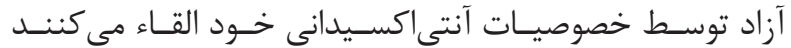

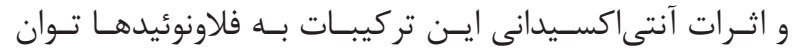

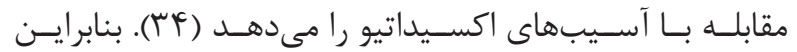

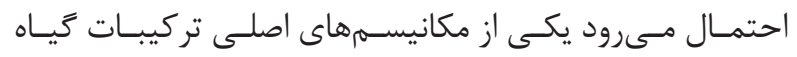

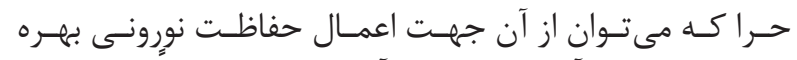

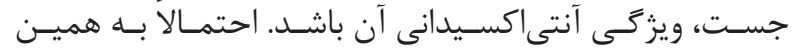

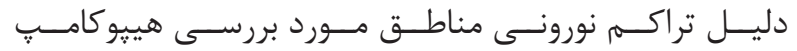

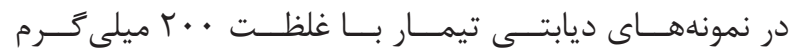

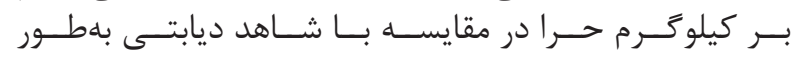

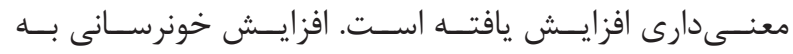

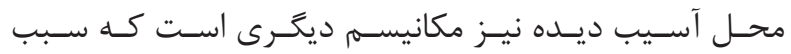

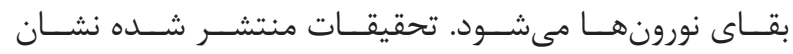

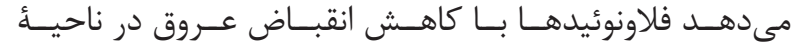

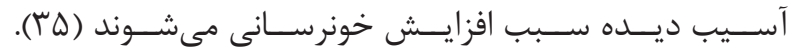

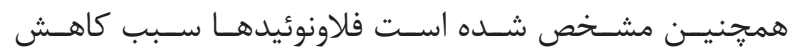

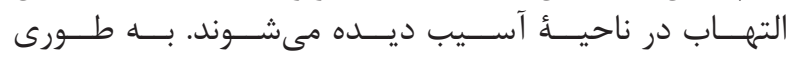

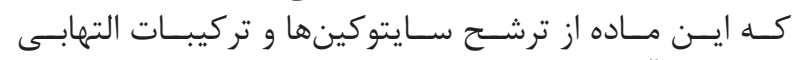

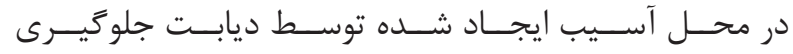

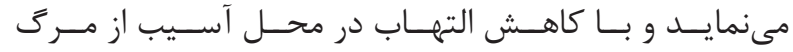

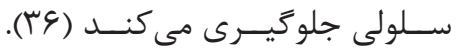

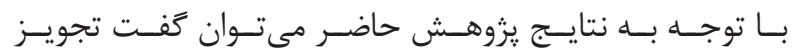

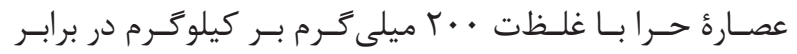

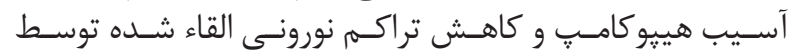

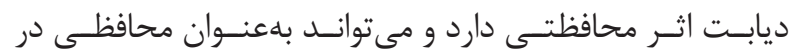

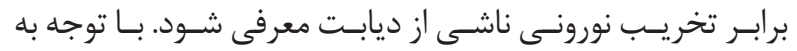

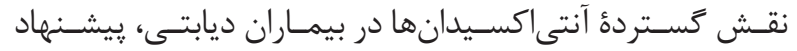

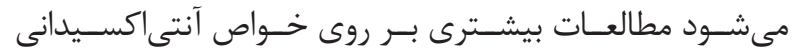

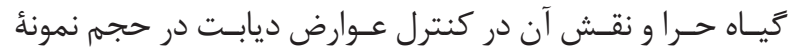

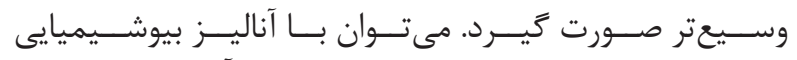

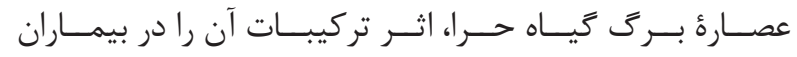

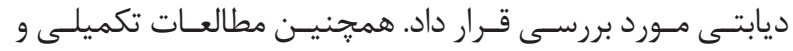

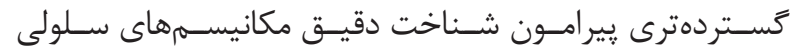

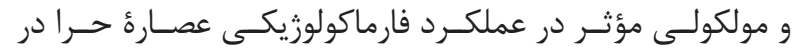

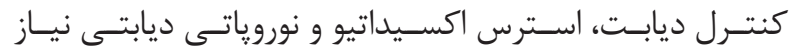

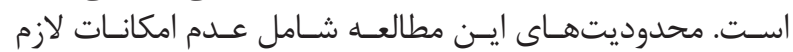

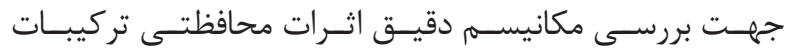

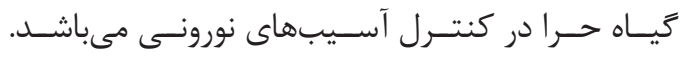

$$
\text { تشكر و قدردانى }
$$

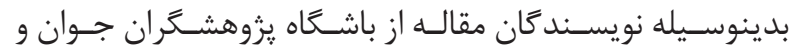

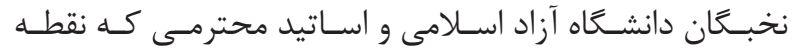

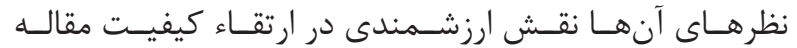

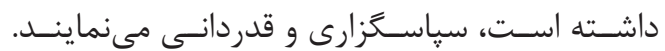

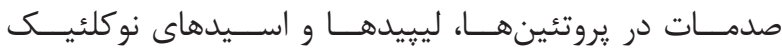

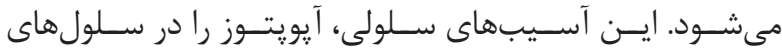

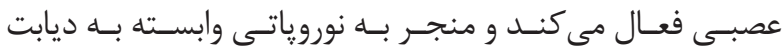

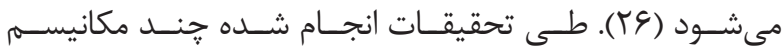

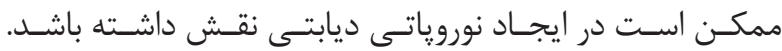

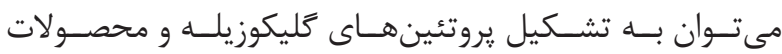

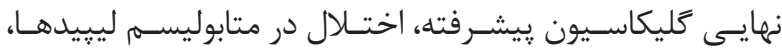

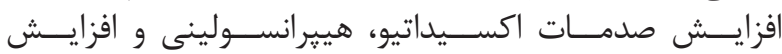

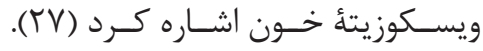

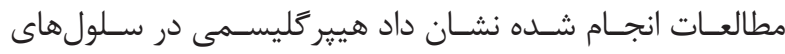

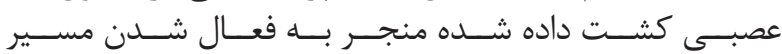

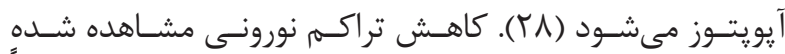

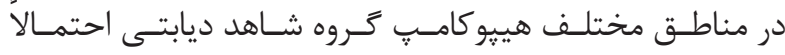

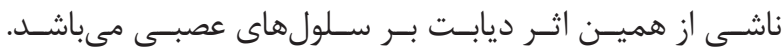

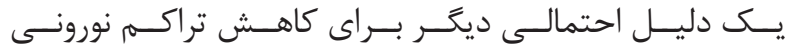

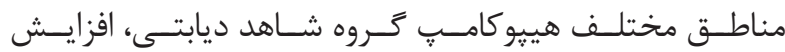

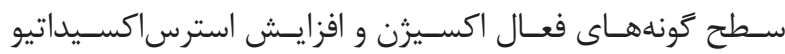

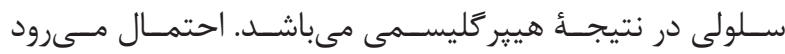

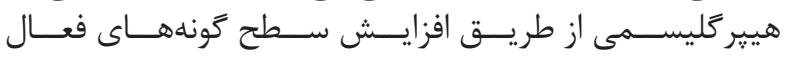

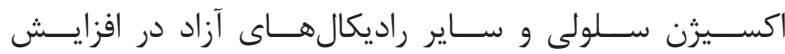

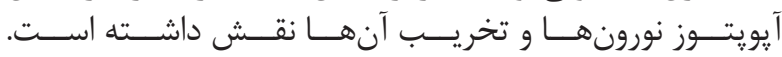

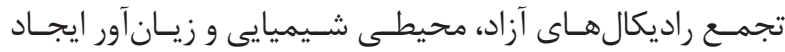

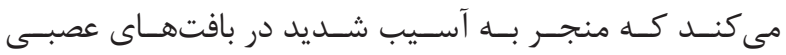
مىشـود (T9).

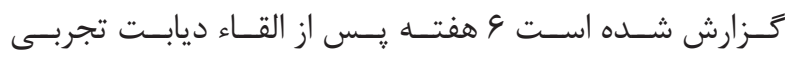

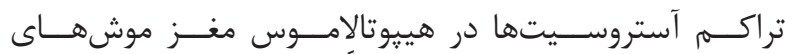

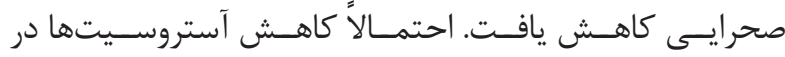

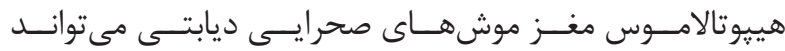

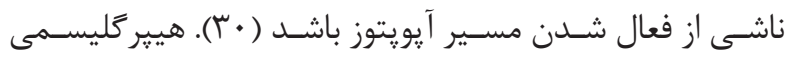

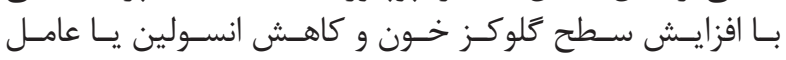

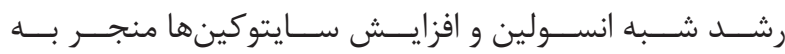

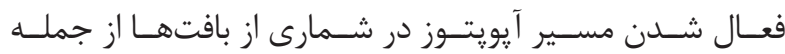

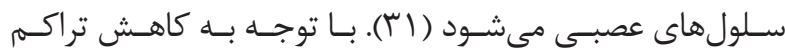

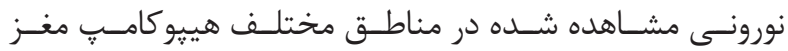

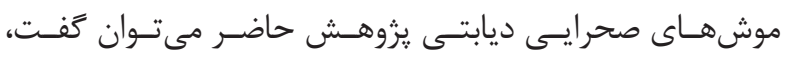

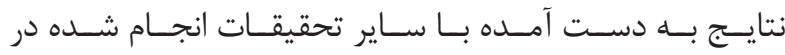

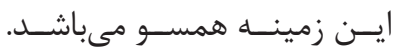

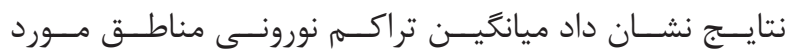

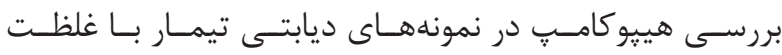

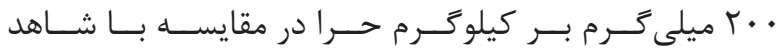

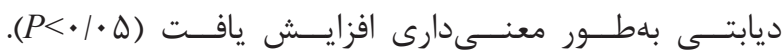

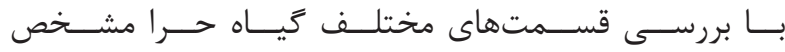

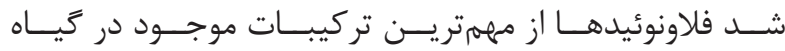

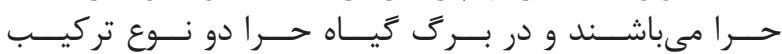

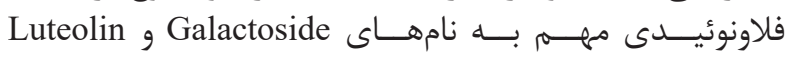
7-O-methylether 39-O-b-D-glucoside

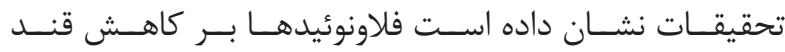

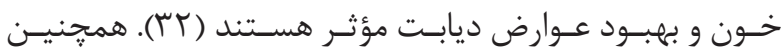


1. Jahanshahi M, Sadeghi Y, Hosseini A, Naghdi N. Change of astrocytes number in CA2 subfield of hippocampus after spatial learning. Armaghan Danesh. 2007; 12(1): 39-48.

2. Grrifine AL. Role of the thalamic nucleus reuniens in mediating interactions between the hippocampus and medial prefrontal cortex during spatial working memory. Front Syst Neurosci. 2015; 9(29). doi: 10.3389/fnsys.

3. Tehranipour M, Kehtarpour M. Effect of alcoholic extract of Cannabis sativa leave on neuronal density of CA1, CA2 and CA 3 regions of rat hippocampus. Feyz. 2012; 16(4): 297-303.

4. Parihar MS, Chaudhary M, Shetty R, Hemnani T. Susceptibility of hippocampus and cerebral cortex to oxidative damage in streptozotocin treated mice: prevention by extracts of Withania somnifera and Aloe vera. J Clin Neurosci. 2004; 11(4): 397-402.

5. King MR, Anderson NJ, Liu C, Law E, Cundiff M, Mixcoatl-Zecuatl TM, et al. Activation of the insulinsignaling pathway in sciatic nerve and hippocampus of type 1 diabetic rats. Neuroscience. 2015; 303: 220-8.

6. Yonguca GN, Dodurgab Y, Adiguzelc E, Gundogdud G, Kucukatayd V, Ozbale S, et al. Grape seed extract has superior beneficial effects than vitamin $\mathrm{E}$ on oxidative stress and apoptosis in the hippocampus of streptozotocin induced diabetic rats. Gene. 2015; 555(2): 119-126.

7. Rodriguez-Martíneza E, Martínezb F, EspinosaGarcíab MT, Maldonadoc P, Rivas-Arancibia S. Mitochondrial dysfunction in the hippocampus of rats caused by chronic oxidative stress. Neuroscience. 2013; 252: 384-95.

8. Lushchak VI. Free radicals, reactive oxygen species, oxidative stress and its classification. Chem Biol Interact. 2014; 224: 164-75.

9. Martinez-Finley EJ, Gavin CE, Aschner M, Gunter TE. Manganese neurotoxicity and the role of reactive oxygen species. Free Radic Biol Med. 2013; 62: 65-75.

10. Patel NT, Gupta A, Pandey AN. Salinity tolerance of Avicennia marina (Forssk.) vierh from gujarat coasts of India. Aquatic Botany. 2010; 93(1): 9-16.

11. Soleimani Z, Mirazi N. The Effect of Avicennia marina hydroethanolic leaf extract on testes tissue and spermatogenesis in male rats induced with carbon tetrachloride. Armaghane-danesh. 2015; 20(8): 677-88.

12. Fathi-Moghaddam H, Mokhtari M, Kamaei L,
Ahangar-pour A. Effects of Avicennia marina leaves aqueous and hydro alcoholic extract on streptozotocininduced diabetic male rats. J Rafsanjan Uni Med Sci. 2011; 10(4): 245-54.

13. Itoigawa $\mathrm{M}$, Ito $\mathrm{C}$, Tan HT-W, Okuda M, Tokuda $\mathrm{H}$, Nishino $\mathrm{H}$, et al. Cancer chemo-preventive activity naphthoquinones and their analogs from Avicennia plants. Cancer letters. 2001; 174: 135-9.

14. Sharaf M, El-Ansari MA, Saleh NAM. New flavonoids from Avicennia marina. Fitoterapia. 2000; 71(3): 274-7.

15. Zamani Gandomani M, Forouzandeh Malati E. Antinociceptive effect of extract of mangrove (Avicennia marina) in male rats. Medical Journal of Tabriz University of Medical Sciences and Health Services. 2014; 36(1): 34-9.

16. Zamani Gandomani M, Forouzandeh Molaali E, Zamani Gandomani Z, Madani H, Jamal Moshtaghian S. Evaluation of anti-inflammatory effect of hydroalcoholic extract of mangrove (Avicennia marina) leaves in male rats. Medical Journal of Tabriz University of Medical Sciences and Health Services. 2012; 34(4): 80-5.

17. Gholami M, Mirazi N. Study of hepato protective effects of Avicennia marina hydro ethanolic leaves extract in male rats induced with carbone tetrachloride. Armaghane Danesh. 2016; 20(10): 858-72.

18. Sepehri-Moghadam H, Rahbarian R, Sadoughi SD. The effect of aqueous extract of Launaea acanthodes (Boiss.) O. Kuntze on the serum level of insulin and blood glucose and histomorphological changes of pancreas in diabetic rats. Feyz. 2015; 19(1): 30-7.

19. Behnam Rasouli M, Nikravesh MR, Mahdavi Shahri N, Tehranipour M. Post-operative time effects after Sciatic nerve crush on the number of Alpha motoneurons, using a stereological counting method (Disector). Iran Biomed J. 2000; 4(1): 45-9.

20. Kamali Ardakani R, Nahangi H, Yadegari M, Hosseini-Sharifabad M. The Effects of long-term administration of methamphetamine on the cerebellum of the male mice: a stereological study. Shefaye Khatam. 2014; 2(4): 37-45.

21. Sahab Negah S, Khaksar Z, Mohammad Sadeghi S, Erfanimajd N, Modarres Mousavi M, Aligholi H, et al. Effect of nettle root extract on histometrical parameters of cerebral and cerebellar cortices in rat following administration of testosterone. Shefaye Khatam. 2015; 3(1): 71-8. 
22. Smith JA, Park S, Krause JS, Banik NL. Oxidative stress, DNA damage, and the telomeric complex as therapeutic targets in acute neurodegeneration. Neurochem Int. 2013; 62(5): 764-75.

23. Verdile G, Fuller SJ, Martins RN. The role of type 2 diabetes in neurodegeneration. Neurobiol Dis. 2015; 84: 22-38.

24. Sadoughi SD, Chamipa M. Effects of aqueous extract of holothuria arenicola and low frequency electromagnetic field on serum insulin, glucose and beta-amyloid (A $\beta 1-42)$ in diabetic rats. Feyz. 2016; 20(1): $1-10$.

25. Majkutewicz I, Kurowska E, Podlacha M, Myślińska D, Grembecka B, Ruciński J, et al. Dimethyl fumarate attenuates intracerebroventricular streptozotocininduced spatial memory impairment and hippocampal neurodegeneration in rats. Behav Brain Res. 2016; 308: 24-37.

26. Cobb CA, Cole MP. Oxidative and nitrative stress in neurodegeneration. Neurobiol Dis. 2015; 84: 4-21.

27. Tesfaye S. Neuropathy in diabetes. Medicine. 2015; 43(1): 26-32.

28. Zhao C, Liu H, Cao R, Ji A, Zhang L, Wang F, et al. Effects of dietary fish oil on learning function and apoptosis of hippocampal pyramidal neurons in streptozotocin-diabetic rats. Brain Res. 2012; 1457: 33-43.

29. Polster BM. AIF, reactive oxygen species, and neurodegeneration: a "complex" problem. Neurochem.
Int. 2013; 62(5): 695-702.

30. Lechuga-Sancho AM, Arroba AI, Frago LM, GarcíaCáceres C, de Célix AD, Argente J, et al. Reduction in the number of astrocytes and their projections is associated with increased synaptic protein density in the hypothalamus of poorly controlled diabetic rats. Endocrinology. 2006; 147(11): 5314-24.

31. Kaboli Kafshgiri S, Ghafari S, Hojjati V, Asadi E, Golalipour MJ. Effect of gestational diabetes on astrocyte density in CA1 and CA3 subfields of hippocampus in rat offspring. J Gorgan Uni Med Sci. 2012; 14(1): 19-25.

32. Yeon JY, Bae YJ, Kim EY, Lee EJ. Association between flavonoid intake and diabetes risk among the koreans. Clinica Chimica Acta. 2015; 15(439): 225-30.

33. Yazhen JC, Jiemin Q, Hong M. Scutellaria flavonoid reduced memory dysfunction and neuronal injury caused by permanent global ischemia in rats. Pharmacology Biochemistry and Behavior. 2005; 82(1): 67-73.

34. Babu PVA, Liu D, Gilbert ER. Recent advances in understanding the anti-diabetic actions of dietary flavonoids. J Nutr Biochem. 2013; 24(11): 1777-89.

35. Lilamand M, Kelaiditi E, Guyonnet S, Antonelli Incalzi R, Raynaud-Simon A, Vellas B, et al. Flavonoids and arterial stiffness: promising perspectives. Nutr Metab Cardiovasc Dis. 2014; 24(7): 698-704.

36. Matias I, Buosi AS, Gomes FCA. Functions of flavonoids in the central nervous system: astrocytes as targets for natural compounds. Neurochem Int. 2016; 95: 85-91. 